\title{
Utilizing high-throughput experimentation to enhance specific productivity of an E.coli T7 expression system by phosphate limitation
}

\author{
Robert Huber ${ }^{*}$, Simon Roth, Natalie Rahmen, Jochen Büchs ${ }^{*}$
}

\begin{abstract}
Background: The specific productivity of cultivation processes can be optimized, amongst others, by using genetic engineering of strains, choice of suitable host/vector systems or process optimization (e.g. choosing the right induction time). A further possibility is to reduce biomass buildup in favor of an enhanced product formation, e.g. by limiting secondary substrates in the medium, such as phosphate. However, with conventional techniques (e.g. small scale cultivations in shake flasks), it is very tedious to establish optimal conditions for cell growth and protein expression, as the start of protein expression (induction time) and the degree of phosphate limitation have to be determined in numerous concerted, manually conducted experiments.

Results: We investigated the effect of different induction times and a concurrent phosphate limitation on the specific productivity of the T7 expression system E.coli BL21(DE3) pRhotHi-2-EcFbFP, which produces the model fluorescence protein EcFbFP upon induction. Therefore, specific online-monitoring tools for small scale cultivations (RAMOS, BioLector) as well as a novel cultivation platform (Robo-Lector) were used for rapid process optimization. The RAMOS system monitored the oxygen transfer rate in shake flasks, whereas the BioLector device allowed to monitor microbial growth and the production of EcFbFP in microtiter plates. The Robo-Lector is a combination of a BioLector and a pipetting robot and can conduct high-throughput experiments fully automated. By using these tools, it was possible to determine the optimal induction time and to increase the specific productivity for EcFbFP from $22 \%$ (for unlimited conditions) to $31 \%$ of total protein content of the E.coli cells via a phosphate limitation.

Conclusions: The results revealed that a phosphate limitation at the right induction time was suitable to redirect the available cellular resources during cultivation to protein expression rather than in biomass production. To our knowledge, such an effect was shown for the first time for an IPTG-inducible expression system. Finally, this finding and the utilization of the introduced high-throughput experimentation approach could help to find new targets to further enhance the production capacity of recombinant E.coli-strains.
\end{abstract}

\section{Background}

One of the major goals in biological process development is to increase the productivity of fermentation processes, in particular, to increase the amount of protein produced per cell (specific productivity). Advantages thereof are usually less effort for product purification, lower fermentation volumes and, thus, smaller bioreactors that are needed to produce a certain amount of product. This can lead to significant cost reductions.

\footnotetext{
*Correspondence: robert.huber@sandoz.com; jochen.buechs@avt.rwthaachen.de

AVT - Aachener Verfahrenstechnik, Biochemical Engineering, RWTH Aachen University, Worringerweg 1, D-52074 Aachen, Germany
}

The specific productivity can be influenced by genetic engineering of strains, choice of the host/vector system (e.g. plasmid copy number and promoter), media optimization or process optimization (e.g. optimizing inducer concentration and induction time; reducing acetate formation by using fed-batch mode) [1]. In particular, the induction time is probably the most crucial factor for inducible recombinant expression systems [2]. Therefore, it is important to screen different induction times, especially if other means of increasing productivity should be evaluated.

Another widespread concept to increase specific productivity is to decouple biomass build-up from product

\section{C) Biomed Central}


formation [3-5]. When recombinant proteins are produced in growing cells, energy and nutritional resources are often directed toward biomass production rather than target protein formation [6]. To circumvent this, the idea is to use cells that are non-growing (dormant) yet are metabolically active and capable of producing protein at higher levels. Rowe and Summers designed such a system (termed Quiescent Cells) using a genetically modified E.coli strain that provides a higher specific productivity by decreasing chromosomal gene expression (and, hence, growth) in favor of plasmidencoded gene expression [6-8]. Another concept is to induce protein expression in the stationary phase of a batch culture [4-6]. Here, the gene of interest is placed under the control of a starvation-inducible promoter that is turned on when glucose becomes exhausted [9]. Compared with exponentially growing cells, stationary phase cells are far more resistent to stress [10,11] and might therefore be suited to produce recombinant proteins [12], as this can place a huge metabolic burden on the cells $[13,14]$. Another starvation-inducible promoter is based on the alkaline phosphatase (phoA) gene. When phosphate is depleted in the medium, the target genes under the control of the phoA promoter are induced $[15,16]$. This system is described for the expression of a number of recombinant proteins $[9,15,17,18]$. A further possibility to limit growth and hereby enhance product formation is to limit secondary substrates such as magnesium, potassium or especially phosphate in the cultivation medium [19].

Phosphate - being one of the most abundant elements in E.coli in terms of the cellular content - plays a crucial role in the cell. Wanner summarized many aspects of the role of phosphate on cell's structure and function [16]. This includes the incorporation of inorganic phosphate in membrane lipids, nucleic acids, carbohydrates and other cellular metabolites. Here, phosphate also acts as an effector of enzymatic reactions [20] and is thus involved in many metabolic pathways, especially in the energy metabolism of the cell (e.g. as high-energy phosphoanhydride bond in ATP). Furthermore, polyphosphate acts as an energy- and phosphate-storing polymer that is used during phosphate limitation $[16,21]$. Because of this universal role of phosphate, a limitation of this nutrient has tremendous effects on the cell growth and physiology. Therefore, many genes are found to be regulated by the level of inorganic phosphate in the medium [22]. A phosphate exhaustion in an E.coli cultivation leads to activation of the Pho regulon [16]. Furthermore, the RpoS response is triggered [23] and cells enter the stationary phase [11]. Due to phosphate limitation, the expression of hundreds of proteins is induced and, at the same time, the levels of more than two hundred proteins are reduced $[10,24]$. In summary, a phosphate limitation leads to quite complex regulatory mechanisms in the cell and is still not yet fully understood on the molecular level.

Nevertheless, phosphate exhaustion has been shown to bolster product formation. This was recently proved in batch cultures for a catabolite-repressed Hansensula polymorpha strain with glucose or glycerol as carbon source [19]. Jensen and Carlsen cultivated an E.coli strain with an constitutive promoter for a recombinant protein in fed-batch mode. By limiting phosphate in the medium they were able to stop the biomass build-up and enhance specific productivity [25]. A similar effect could be achieved for producing shikimic acid in E.coli in a phosphate-limited chemostat culture [26] and for lysine production with Corynebacterium glutamicum [27]. However, besides using the phoA promoter, there are only few reports on phosphate-limited batch cultivations of E. coli [26]. Moreover, there are no reports specifically on using phosphate limitation with the most common inducible promoter system, i.e. the T7 or lac promoter, to enhance specific productivity of recombinant protein expression. The reason for this might be the difficulty to establish optimal conditions for inducing protein expression in phosphate-limited cultures. For this purpose, many experiments have to be conducted in parallel batch cultures to determine the best phosphate content of the medium and the optimal time of induction. So far, such studies are very tedious with conventional means (e.g. shake flask or microtiter plates (MTPs) experiments with offline-measurement of growth and manual induction of protein expression at an appropiate time).

Hence, the aim of this work was to investigate different induction times and in particular to increase specific productivity of a T7 expression system via phosphate limitation. Therefore, the influence of phosphate on growth and recombinant protein expression (at various induction times) in batch cultures was studied with specific online-monitoring tools for small scale cultivations, namely RAMOS [28,29] and BioLector [30,31]. Furthermore, a novel cultivation platform (termed Robo-Lector) was applied [32]. This system conducts high-throughput experiments in MTPs automatically and allows to monitor microbial growth and the production of fluorescence proteins online.

\section{Methods}

\section{Microorganism}

For all experiments the strain E.coli BL21(DE3) pRhotHi-2-EcFbFP was used (kindly provided by $\mathrm{T}$. Drepper, Institute of Molecular Enzyme Technology, Heinrich-Heine-University Düsseldorf, Germany) [33]. The used expression plasmid harbors a kanamycin resistence gene and the $\mathrm{T} 7$ promoter that is under the 
control of the lac operator. The fluorescent protein EcFbFP is expressed as a model protein. Therefore, the EcFbFP encoding gene was cloned into the pRhotHi-2 vector downstream of the $\mathrm{T} 7$ promoter. $\mathrm{A} \mathrm{His}_{6}$-tag was fused to the $\mathrm{C}$-terminus of the EcFbFP resulting in a recombinant fusion protein with a molecular weight of $16.5 \mathrm{kDa}$. The used EcFbFP is also available under the trademark evoglow (evocatal GmbH, Düsseldorf, Germany).

\section{Media and Solutions}

For all precultures TB-Medium was used, consisting of $24 \mathrm{~g} / \mathrm{L}$ yeast extract, $12 \mathrm{~g} / \mathrm{L}$ tryptone, $12.54 \mathrm{~g} / \mathrm{L}$ $\mathrm{K}_{2} \mathrm{HPO}_{4}, 2.31 \mathrm{~g} / \mathrm{L} \mathrm{KH}_{2} \mathrm{PO}_{4}$ and $5 \mathrm{~g} / \mathrm{L}$ glycerol. The medium was supplemented with kanamycin $(50 \mu \mathrm{g} / \mathrm{L})$ and the $\mathrm{pH}$ was adjusted to 7.2 with $\mathrm{NaOH}$.

All main cultivations were performed in a modified form of Wilms \& Reuss synthetic medium [34,35]. The medium consists of $7.5 \mathrm{~g} / \mathrm{L}$ glucose, $5 \mathrm{~g} / \mathrm{L}\left(\mathrm{NH}_{4}\right)_{2} \mathrm{SO}_{4}$, $0.5 \mathrm{~g} / \mathrm{L} \mathrm{NH}_{4} \mathrm{Cl}, 3 \mathrm{~g} / \mathrm{L} \mathrm{K}_{2} \mathrm{HPO}_{4}$ (for phosphate unlimited conditions), $2 \mathrm{~g} / \mathrm{L} \mathrm{Na}_{2} \mathrm{SO}_{4}, 0.5 \mathrm{~g} / \mathrm{L} \mathrm{MgSO}_{4} \cdot 7 \mathrm{H}_{2} \mathrm{O}, 41.85$ g/L 3-(N-Morpholino)-propanesulfonic acid (MOPS), $0.01 \mathrm{~g} / \mathrm{L}$ thiamine hydrochloride, $1 \mathrm{~mL} / \mathrm{L}$ trace element solutions. For determining the phosphate demand of the applied strain, different media were prepared with the following phosphate concentrations (as $\mathrm{K}_{2} \mathrm{HPO}_{4}$ in $\mathrm{mg} /$ L): 3000, 750, 300, 225, 195, 180, 150, 105, 90, 75, 60, 45 , 30. All media preparations were supplemented with kanamycin $(50 \mu \mathrm{g} / \mathrm{L})$ and the $\mathrm{pH}$ was adjusted to 7.5 with $\mathrm{NaOH}$.

For induction of protein expression a sterile-filtered IPTG stock solution $(100 \mathrm{mM})$ was used to give a final IPTG concentration of $0.1 \mathrm{mM}$ in the cultures. This concentration was identified in previous experiments to be suitable for efficient recombinant protein expression (data not shown). All reagents were of analytical grade and supplied by Carl Roth GmbH \& Co. KG (Karlsruhe, Germany).

\section{Cultivation}

\section{Preculture}

The precultures for all experiments were inoculated from a cryoculture of E.coli BL21(DE3) pRhotHi-2EcFbFP and cultivated in a $250 \mathrm{~mL}$ Erlenmeyer shake flask with $10 \mathrm{~mL}$ TB-medium. The flask was placed on a rotary shaker at a shaking frequency (n) of $300 \mathrm{rpm}$, a shaking diameter $\left(\mathrm{d}_{0}\right)$ of $50 \mathrm{~mm}$ and a temperature (T) of $37^{\circ} \mathrm{C}$ for $16 \mathrm{~h}(\mathrm{t})$. To ensure that no disturbing media components from the preculture medium - especially phosphate - influence the limitation experiments, the harvested cells were washed twice. Therefore, cells of the preculture were pelleted by centrifugation at 4000 rpm and resuspended in phosphate-free modified Wilms \& Reuss medium. After two washing steps, the subsequent main cultures were inoculated to give an initial optical density (OD) of 0.4. OD was measured at 600 nm with a Uvikon 922 spectrophotometer (Kontron, Milano, Italy).

\section{Microtiter plate cultivations}

Cultivations in MTPs were conducted in a BioLector $[30,31]$ which is a system that can permanently monitor microbial growth and the fluorescence of reporter proteins under defined conditions in a MTP during the cultivation. Therefore, a MTP with medium is inoculated and placed in the incubation chamber of the BioLector (Figure 1A). The MTP is continuously shaken to provide a sufficient oxygen transfer to the culture. For growth and product monitoring, light with a defined wavelength is sent into each well (excitation), whereas the backscattered light (indicator for biomass) or fluorescence

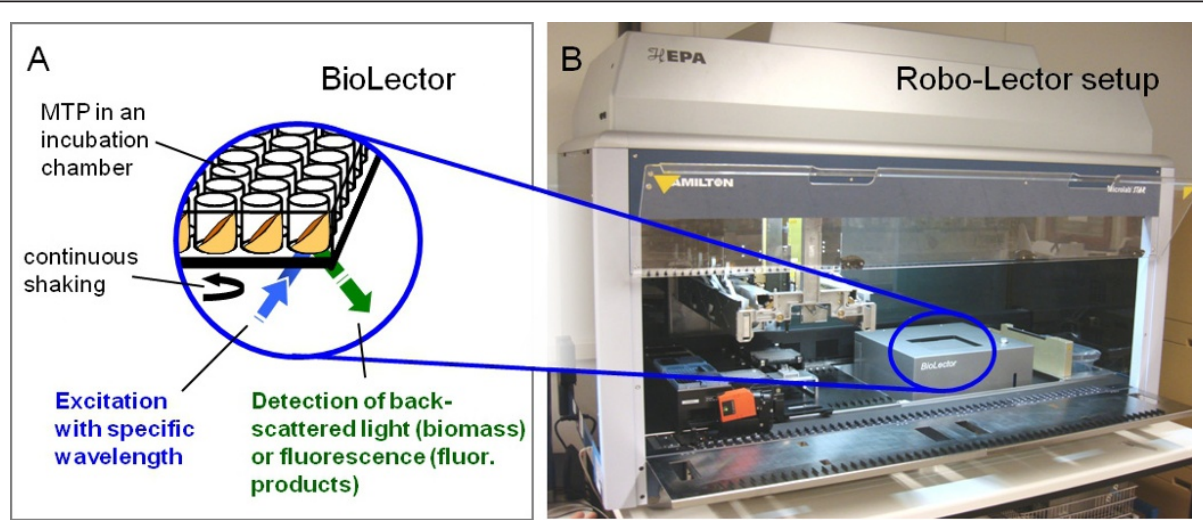

Figure 1 Measurement principle of the BioLector (A) and experimental setup for the Robo-Lector (B). The BioLector provides a system for cultivating microorganisms in a microtiter plate (MTP) inside an incubation chamber. The MTP is continuously shaken to provide sufficient oxygen to the culture. An optical measurement system continuously monitors biomass concentration and fluorescent products. The Robo-Lector setup consists of a BioLector integrated into a pipetting robot, enabling fully automated high throughput cultivation experiments in a MTP format. 
(indicator for fluorescent products) is detected and analysed. In this study, the experiments to determine the phosphate demand and the influence of phosphate limitation on protein production were conducted in a BioLector prototype [30].

The screening for the optimal induction time was carried out with the Robo-Lector platform described by Huber et al. [32]. The Robo-Lector platform consists of a BioLector (m2p-labs, Aachen, Germany) [31] integrated into a pipetting robot (Figure 1B). This system enables one to conduct automated high-throughput cultivations. Here, the method 'induction profiling' was used, which performs an automated induction of parallel cultures by the pipetting of IPTG-solutions in the respective wells of a main culture MTP at different times. For a detailed description of the method please refer to Huber et al. (2009). The method was used with the following modifications: the induction time was varied from 1 to $10 \mathrm{~h}$ and the inducer concentration was kept constant at $0.1 \mathrm{mM}$. The following conditions were applied for all cultivations in the BioLector prototype and the Robo-Lector: $37^{\circ} \mathrm{C}$, shaking diameter $3 \mathrm{~mm}$, shaking frequency $950 \mathrm{rpm}$. All cultivations were performed in sterile black 96 well MTP ( $\mu$ Clear, Cat. 655087, Greiner Bio-One, Frickenhausen, Germany) with an initial filling volume of $200 \mu \mathrm{L}$ (BioLector prototype) or $190 \mu \mathrm{L}$ (Robo-Lector). To avoid evaporation, each utilized MTP were sealed with a gas-permeable adhesive seal (ThermoScientific, Waltham, USA) for the cultivations in the BioLector prototype. For the RoboLector experiments, the MTP was sealed with sterile pierceable, resealable tape (X-Pierce, Excel Scientific, Victorville, USA), allowing ventilation of the wells at reduced evaporation rates. The EcFbFP fluorescence was monitored at an excitation wavelength of $460 \mathrm{~nm}$ and an emission wavelength of $492 \mathrm{~nm}$. The resulting fluorescence is a suitable quantitative signal for in-vivo EcFbFP expression [36,37] and therefore corresponds to the total volumetric yield of the target protein EcFbFP. The biomass concentration was measured via scattered light intensity (I) and was detected at an excitation wavelength of $620 \mathrm{~nm}$. The initial scattered light intensity $\left(\mathrm{I}_{0}\right)$ was mainly attributed to factors such as the media background or the type of the MTP and thus was substracted from the residual scattered light data $\left(I-I_{0}\right)$ [30] or fluorescence data (where appropiate). Different signal gain factors for scattered light and fluorescence were used as stated in the figure captions. The specific productivity for EcFbFP expression in the MTP cultivations was defined as EcFbFP fluorescence per scattered light intensity (given in a.u.).

\section{RAMOS cultivations}

The RAMOS device provides analysis of microbial growth in shake flasks by measuring the respiratory activity of the microorganisms $[28,29,38]$. The respiration activity (Oxygen Transer Rate, OTR) in different phosphate-containing cultures was measured with the RAMOS device in modified $250 \mathrm{~mL}$ Erlenmeyer shake flasks with a filling volume of $10 \mathrm{~mL}$. Shake flasks with $3000 \mathrm{mg} / \mathrm{L}, 150 \mathrm{mg} / \mathrm{L}$ and $75 \mathrm{mg} / \mathrm{L} \mathrm{K}_{2} \mathrm{HPO}_{4}$ were cultured in duplicate. All cultivations were carried out with a shaking frequency of $350 \mathrm{rpm}$ and at a shaking diameter of $50 \mathrm{~mm}$ on a Lab-Shaker LS-K (Kühner, Birsfelden, Switzerland) at $\mathrm{T}=37^{\circ} \mathrm{C}$. To determine $\mathrm{PO}_{4}{ }^{3-}$, glucose and acetate concentrations, separate shake flasks were cultured in a Lab-Shaker LS-K under identical conditions as in the RAMOS device. From these shake flasks samples were collected approximately every $3 \mathrm{~h}$. Phosphate $\left(\mathrm{PO}_{4}{ }^{3-}\right)$ was analysed using a photometric kit (Spectroquant, Cat. 1.00616.0001, Merck, Darmstadt, Germany). $\mathrm{PO}_{4}{ }^{3-}$ has a molar fraction of $55 \%$ in $\mathrm{K}_{2} \mathrm{HPO}_{4}$, hence $3000 \mathrm{mg} / \mathrm{L} \mathrm{K}_{2} \mathrm{HPO}_{4}$ correspond to 1650 $\mathrm{mg} / \mathrm{L} \mathrm{PO}_{4}{ }^{3-}, 150 \mathrm{mg} / \mathrm{L} \mathrm{K}_{2} \mathrm{HPO}_{4}$ correspond to $83 \mathrm{mg} / \mathrm{L}$ $\mathrm{PO}_{4}{ }^{3-}$ and $75 \mathrm{mg} / \mathrm{L} \mathrm{K}_{2} \mathrm{HPO}_{4}$ correspond to $41 \mathrm{mg} / \mathrm{L}$ $\mathrm{PO}_{4}{ }^{3-}$. Glucose and acetate were measured in duplicate via HPLC (Ultimate 3000, Dionex, Sunnyvale, USA) on an organic acid resin (CS-Chromatographie Service, Langerwehe, Germany) at $60^{\circ} \mathrm{C}$ with $5 \mathrm{mM} \mathrm{H}_{2} \mathrm{SO}_{4}$ as eluent.

\section{Shake flask cultivations}

Shake flask experiments were conducted to verify results from the MTP scale. These cultures were grown in 250 $\mathrm{mL}$ Erlenmeyer shake flasks which were filled with 10 $\mathrm{mL}$ modified Wilms \& Reuss medium with different phosphate concentrations $(3000,225,150,75 \mathrm{mg} / \mathrm{L}$ $\mathrm{K}_{2} \mathrm{HPO}_{4}$ ) at a shaking frequency of $350 \mathrm{rpm}$, a shaking diameter of $50 \mathrm{~mm}$ and $37^{\circ} \mathrm{C}$. The cultures were induced with IPTG (final concentration of $0.1 \mathrm{mM}$ ) after $6 \mathrm{~h}$ of growth. After 26 hours, the OD of the cultures was measured and cells were harvested to perform product analysis via SDS-PAGE. Furthermore, scattered light and fluorescence intensities of culture samples $(200 \mu \mathrm{L})$ were measured in the BioLector prototype.

\section{SDS-PAGE and densitometry}

SDS-PAGE was performed to correlate the specific productivities of the shake flask cultivations with different phosphate concentrations. The SDS-PAGE was conducted with the XCell SureLock MiniCell (Invitrogen, Carlsbad, USA) and a Bis-tris-gel (NuPAGE 4-12\%, Invitrogen) according to the manufacturer's instructions. Normalized samples were prepared by suspending equal amounts of cells from the shake flask experiments in SDS-buffer and loading them on the gel. Additionally, a molecular weight standard (RotiMark, Roth, Crailsheim, Germany) was loaded on the gel. After electrophoresis, the gel was stained and destained according to the manufacturer's instructions (SimplyBlue SafeStain, 
Invitrogen). The destained gel was photographed with the documentation system ChemiDoc (Biorad, Hercules, USA) and analyzed with the densitometry computer program Total Lab TL100 (Nonlinear Dynamics, Newcastle upon Tyne, UK) to determine the specific productivity of the different samples. The specific productivity was defined as the ratio of the density of the target protein band (EcFbFP) to the total density of all bands of the corresponding sample (band\%).

\section{Results and Discussion}

\section{Effects of phosphate limitation on growth}

The aim of this study was to examine the effects of a phosphate limitation on the growth of the E.coli strain BL21(DE3) pRhotHi-2-EcFbFP and its recombinant product formation. First of all, the growth behavior at different phosphate concentrations was investigated in the BioLector device. A graduated growth pattern resulted from this experiment (Figure 2A). When no phosphate was added to the medium $(0 \mathrm{mg} / \mathrm{L})$ there was nevertheless some growth visible. This might be explained by some residual phosphate from the preculture (though these cells were washed two times with phosphate-free medium) and most likely because of intracellular polyphosphate stores which were mobilized during phosphate starvation [11]. The phosphate content of the original medium was $3000 \mathrm{mg} / \mathrm{L}$, and this culture showed a typical batch culture curve with exponential growth. The stationary phase began at approximately 13 $\mathrm{h}$ with a biomass concentration of 30000 a.u. (Figure 2A). This culture was phosphate unlimited, whereas the cultures with 300 and $750 \mathrm{mg} / \mathrm{L}$ reached a slightly higher scattered light intensity. This could be explained by the fact that excess phosphate in the medium (3000 $\mathrm{mg} / \mathrm{L}$ ) could form a complex with trace elements (unpublished results) and this, in turn, led to a slightly lower biomass yield compared to 300 or $750 \mathrm{mg} / \mathrm{L}$ phosphate. The cultures with less than $300 \mathrm{mg} / \mathrm{L}$ phosphate showed lower biomass yields as phosphate became more and more limited. This correlation can also be seen in Figure 2B, where an almost linear relationship between biomass yield (scattered light intensity at the end of the cultivation) and phosphate content of the medium becomes apparent. From such a depiction, the minimal phosphate demand of the cells for unlimited growth (here: $300 \mathrm{mg} / \mathrm{L}$ ) could easily be determined [39]. All cultures with phosphate showed the same increase in scattered light intensities in the beginning (Figure 2A), indicating a comparable maximum specific growth rate $\left(\mu_{\max }=0.79 \pm 0.09\right)$. As soon as phosphate became limited, these cultures deviated from the unlimited cultures and then continued with slow growth until they reached a plateau (Figure 2A). This slight increase in biomass concentration had been shown elsewhere

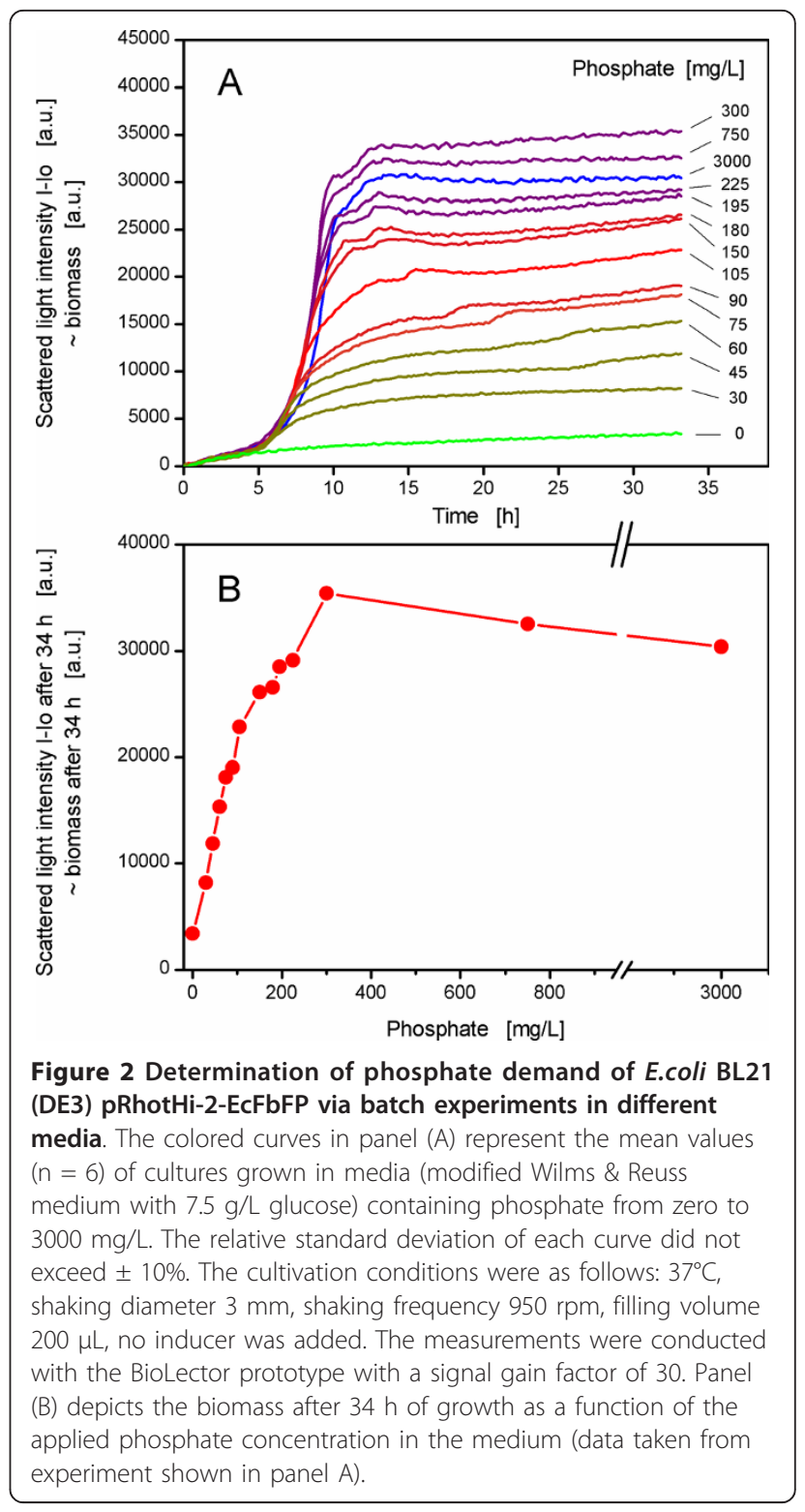

$[11,18,40]$ and seemed typical because of the metabolism of intercellular phosphate reserves. With the approach to monitor growth in MTPs, the demand of the examined E.coli strain for phosphate could be investigated in detail. Futhermore, the time when phosphate became limited was derived from the online-monitored growth curves.

In order to verify these results, a cultivation in the RAMOS system was conducted with three selected phosphate concentrations (Figure 3A). Derived from the results of Figure 2, a culture with $3000 \mathrm{mg} / \mathrm{L}$ dipotassium phosphate was chosen as reference for phosphate unlimited conditions. Besides that, cultivations with 150 $\mathrm{mg} / \mathrm{L}$ and $75 \mathrm{mg} / \mathrm{L} \mathrm{K}_{2} \mathrm{HPO}_{4}$ were conducted to represent intermediate and strong phosphate limitations, 

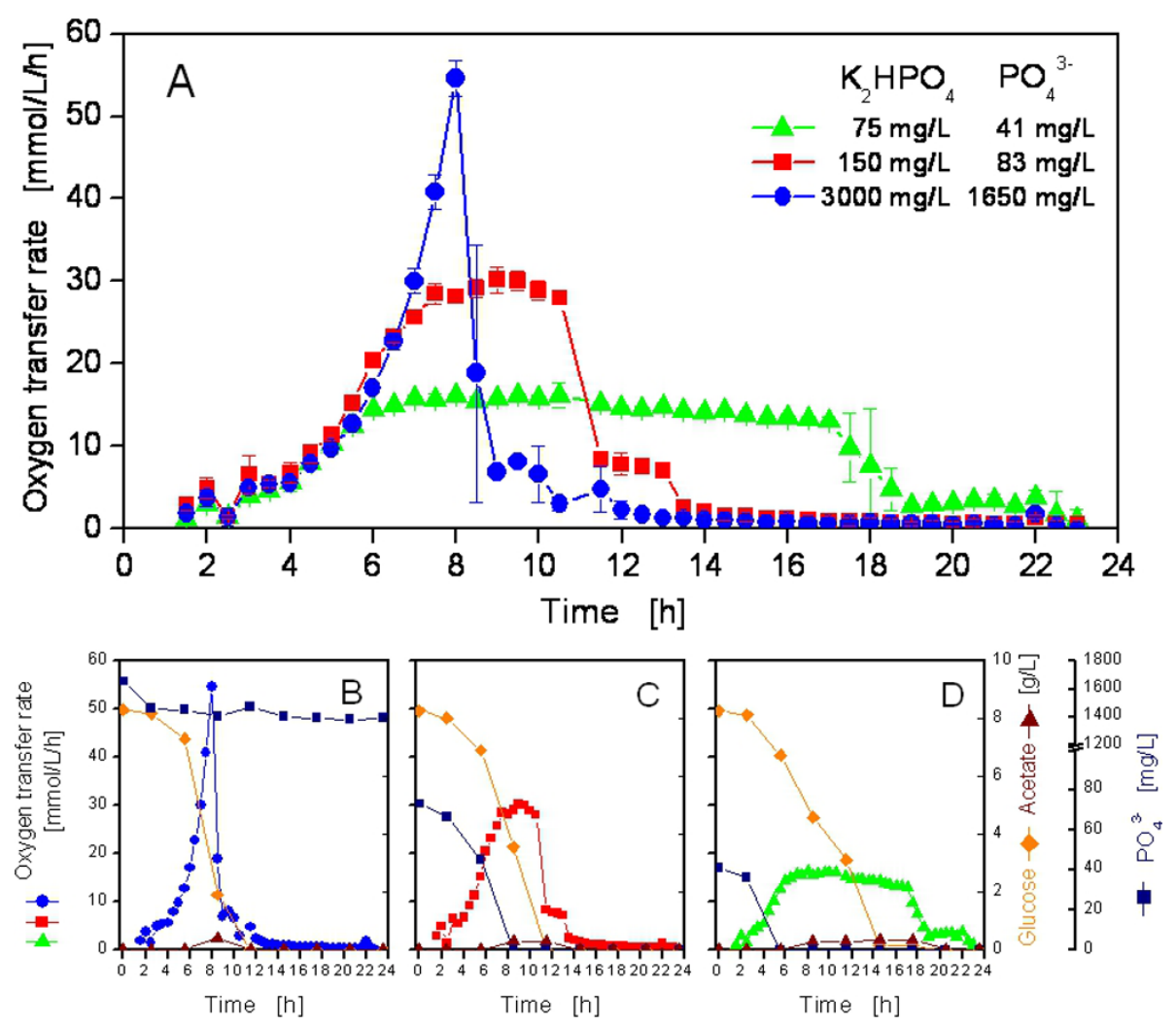

Figure 3 Influence of phosphate limitation on respiration activity during cultivation. The oxygen transfer rate (OTR) was measured with the RAMOS device. In panel $(\mathrm{A})$, the mean $(\mathrm{n}=2)$ and standard deviations of duplicate cultivations with $3000 \mathrm{mg} / \mathrm{L} \mathrm{K}_{2} \mathrm{HPO}_{4}(\cdot, \mathrm{blue}), 150 \mathrm{mg} / \mathrm{L}$ $\mathrm{K}_{2} \mathrm{HPO}_{4}\left(\boldsymbol{\bullet}\right.$, red) and $75 \mathrm{mg} / \mathrm{L} \mathrm{K}_{2} \mathrm{HPO}_{4}(\boldsymbol{\bullet}$, green) are depicted. In separate shake flasks, samples were collected and analysed for glucose, acetate and phosphate $\left(\mathrm{PO}_{4}{ }^{3-}\right)$. The results of these analyses and the OTR are depicted in (B) for $3000 \mathrm{mg} / \mathrm{L}$, in (C) for $150 \mathrm{mg} / \mathrm{L}$ and in (D) for $75 \mathrm{mg} / \mathrm{L}$ $\mathrm{K}_{2} \mathrm{HPO}_{4}$. The cultivation conditions were as follows: $37^{\circ} \mathrm{C}$, shaking diameter $50 \mathrm{~mm}$, shaking frequency $350 \mathrm{rpm}$, filling volume $10 \mathrm{~mL}$, no induction; modified Wilms \& Reuss medium (7.5 g/L Glucose) with various phosphate contents.

respectively. The unlimited culture showed a typical exponential growth reaching a maximal OTR of 55 $\mathrm{mmol} / \mathrm{L} / \mathrm{h}$ after $8 \mathrm{~h}$. At this time, the OTR decreased sharply, showing a second small peak at 9 to $10 \mathrm{~h}$. This behavior is typical for an E.coli batch cultivation where the primary carbon source, namely glucose, became exhausted (after 8 to $9 \mathrm{~h}$ ) and the overflow metabolite acetate was consumed subsequently (after 9 to $11 \mathrm{~h}$, Figure 3B). Although glucose, acetate and phosphate were not measured in shorter intervals, numerous studies dealing with the interpretation of OTR curves for microbial cultivations affirm this interpretation (e.g. $[19,41,42])$. Phosphate became not limited with 3000 $\mathrm{mg} / \mathrm{L} \mathrm{K}_{2} \mathrm{HPO}_{4}$ (Figure 3B). The OTR of the limited culture with $150 \mathrm{mg} / \mathrm{L} \mathrm{K}_{2} \mathrm{HPO}_{4}$ started to stagnate at an OTR of $30 \mathrm{mmol} / \mathrm{L} / \mathrm{h}$ after 6 to $7 \mathrm{~h}$ when phosphate limitation began (Figure 3C). After $11 \mathrm{~h}$, the OTR dropped again, because glucose was exhausted. This culture showed a second short OTR plateau at about $12 \mathrm{~h}$, mainly because of the metabolism of the hitherto produced acetate. The $75 \mathrm{mg} / \mathrm{L}$-culture (Figure 3D) showed a similar pattern, although the OTR reached only 17 $\mathrm{mmol} / \mathrm{L} / \mathrm{h}$ at its maximum and slowly decreased until glucose was exhausted after about 15 to $17 \mathrm{~h}$. This behavior is typical for a limitation of secondary substrates such as phosphate $[19,28]$. The culture started phosphate-limited growth after 5 to $6 \mathrm{~h}$ and showed a second small OTR plateau after $19 \mathrm{~h}$, obviously because the residual amounts of acetate or other, not measured by-products were consumed. These findings, especially the times when phosphate limitation started and glucose was exhausted, are in good agreement with the growth data from the BioLector (Figure 2A).

The above results clearly showed that after a limitation of phosphate, the cells stagnated in growth and exhibited a prolonged respiration activity due to the ongoing consumption of the primary substrate (glucose) for maintenance. Similar results have been found with extensive offline analysis of growth, phosphate and glucose in a bioreactor $[18,40]$. The measurement of the respiration activity provided valueable insight into the metabolism of the cells and confirmed the proposed 
events during phoshate limitation. The period of a stagnating OTR depicted in Figure 3 (phosphate-limited cultures) represented a time of metabolic activity yet reduced growth of the cells and might be used to decouple recombinant protein expression from biomass growth.

\section{Effects of phosphate limitation on protein expression}

A BioLector experiment was conducted to study in detail the general effects of a phosphate limitation on the expression of the fluorescence protein EcFbFP. Therefore, an intermediate phosphate concentration $(150 \mathrm{mg} / \mathrm{L})$ was chosen. Besides this, also the unlimited medium was used as a reference. From the results of Figure 2 and 3 an induction time of 9 to $10 \mathrm{~h}$ was chosen because at that time the phosphate became limited (for the $150 \mathrm{mg} / \mathrm{L}$-culture). The unlimited $3000 \mathrm{mg} / \mathrm{L}$ culture grew to a scattered light intensity of 19900 a.u. (values rounded to the nearest 100), whereas the 150 $\mathrm{mg} / \mathrm{L}$-culture reached only 13100 a.u. (Figure $4 \mathrm{~A}$ ). The unlimited culture immediately started to express the fluorescent protein EcFbFP upon induction and reached a plateau at a fluorescence of 2400 a.u. (Figure 4B).

After this culture consumed all the glucose, it reached stationary phase $(13 \mathrm{~h})$ and the cells had no longer the resources (substrate, energy) to express the target protein. On the other hand, the limited culture exhibited a much greater EcFbFP fluorescence (6900 a.u.) upon induction, even when the important nutrient phosphate was limited (Figure 4B). The production of EcFbFP continued until $14 \mathrm{~h}$ (probably because glucose was exhausted as shown in Figure 3 for an non-induced culture), though the cells did not grow anymore. Although a lower biomass was available in the phosphate-limited culture, even more EcFbFP was produced than in the unlimited culture. Accordingly, the specific productivity (EcFbFP fluorescence per scattered light intensity) at the end of the cultivation was higher for limited conditions (0.53 a.u.) than for the unlimited culture (0.12 a.u.). This provided evidence that phosphate-limited cells are still capable to produce recombinant proteins. The phosphate unlimited culture in this experiment was induced almost in the stationary phase and hence had only little resources left for efficient EcFbFP expression. Furthermore, the time of induction is probably the most crucial factor for recombinant protein expression [2]. Therefore, it is important to screen at different induction times for phosphate-limited and -unlimited cultures to evaluate if a phosphate limitation is generally suitable to enhance specific productivity.

For such a screening, a novel cultivation platform for automated high-throughput experimentation [32] was used. This system consists of a BioLector integrated into a liquid handling workstation. With this platform, it is

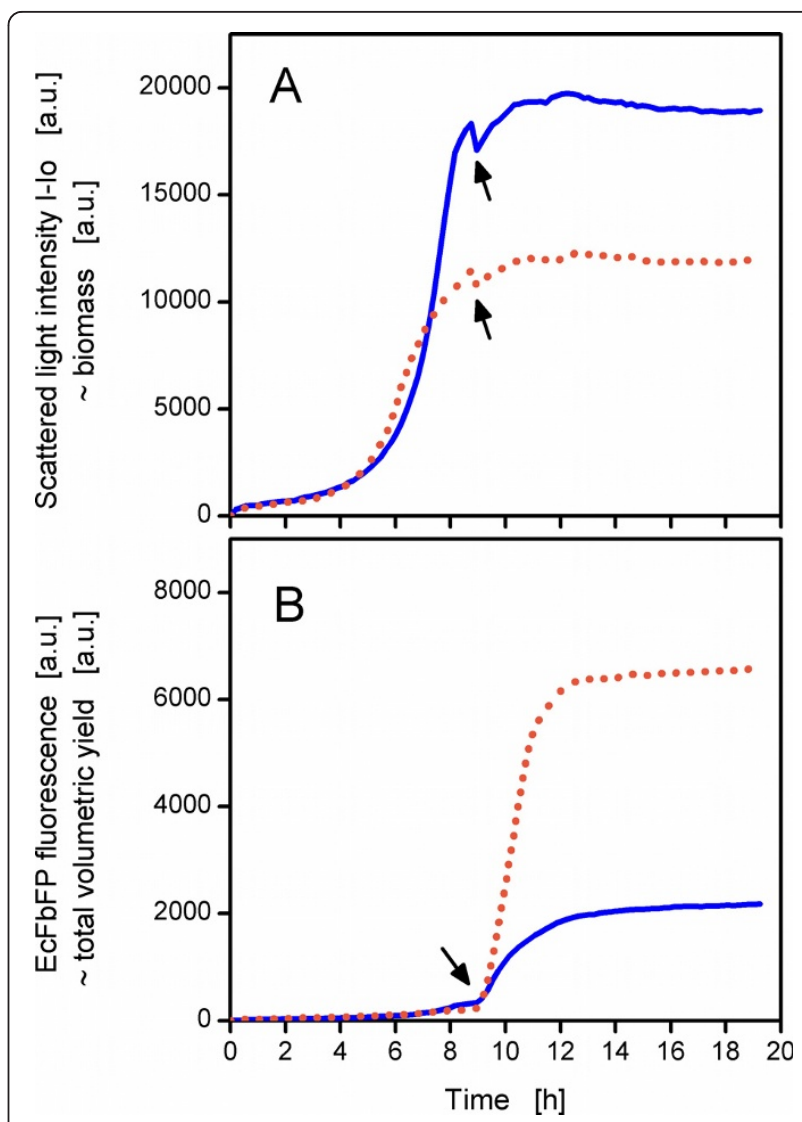

Figure 4 Influence of phosphate limitation on growth (A) and recombinant protein production $(B)$. The measurements (scattered light as an indicator for biomass, panel (A); EcFbFP fluorescence as an indicator for total volumetric yield, panel (B)) were conducted in the BioLector prototype with a signal gain factor of 25. Each of the four curves shown represent the mean values ( $n$ $=12$ ) of cultures grown in media (modified Wilms \& Reuss media with $7.5 \mathrm{~g} / \mathrm{L}$ glucose) containing phosphate at two different concentrations: $3000 \mathrm{mg} / \mathrm{L} \mathrm{K}_{2} \mathrm{HPO}_{4}$ (unlimited conditions, solid blue lines) and $150 \mathrm{mg} / \mathrm{L} \mathrm{K}_{2} \mathrm{HPO}_{4}$ (phosphate-limited conditions, dotted orange lines). The relative standard deviation of each curve did not exceed $\pm 10 \%$. The cultivation conditions were as follows: $37^{\circ} \mathrm{C}$, shaking diameter $3 \mathrm{~mm}$, shaking frequency $950 \mathrm{rpm}$, filling volume $200 \mu \mathrm{L}$, induction with IPTG (final concentration $0.1 \mathrm{mM}$ ) after $9.5 \mathrm{~h}$ (indicated by arrows)

possible to automatically induce up to 96 parallel cultures in a MTP at different times. This method, called 'induction profiling' [32], was modified here to test 9 different induction times (at a constant IPTG concentration of $0.1 \mathrm{mM})$ for phosphate-limited $(150 \mathrm{mg} / \mathrm{L})$ or unlimited cultures $(3000 \mathrm{mg} / \mathrm{L})$.

Figure 5A depicts the EcFbFP expression in phosphate unlimited cultures at different induction times. As a reference, cells without induction were cultivated and showed only small background fluorescence. At every induction point (from 1 to $9 \mathrm{~h}$ after inoculation), an immediate increase of the EcFbFP fluorescence became 


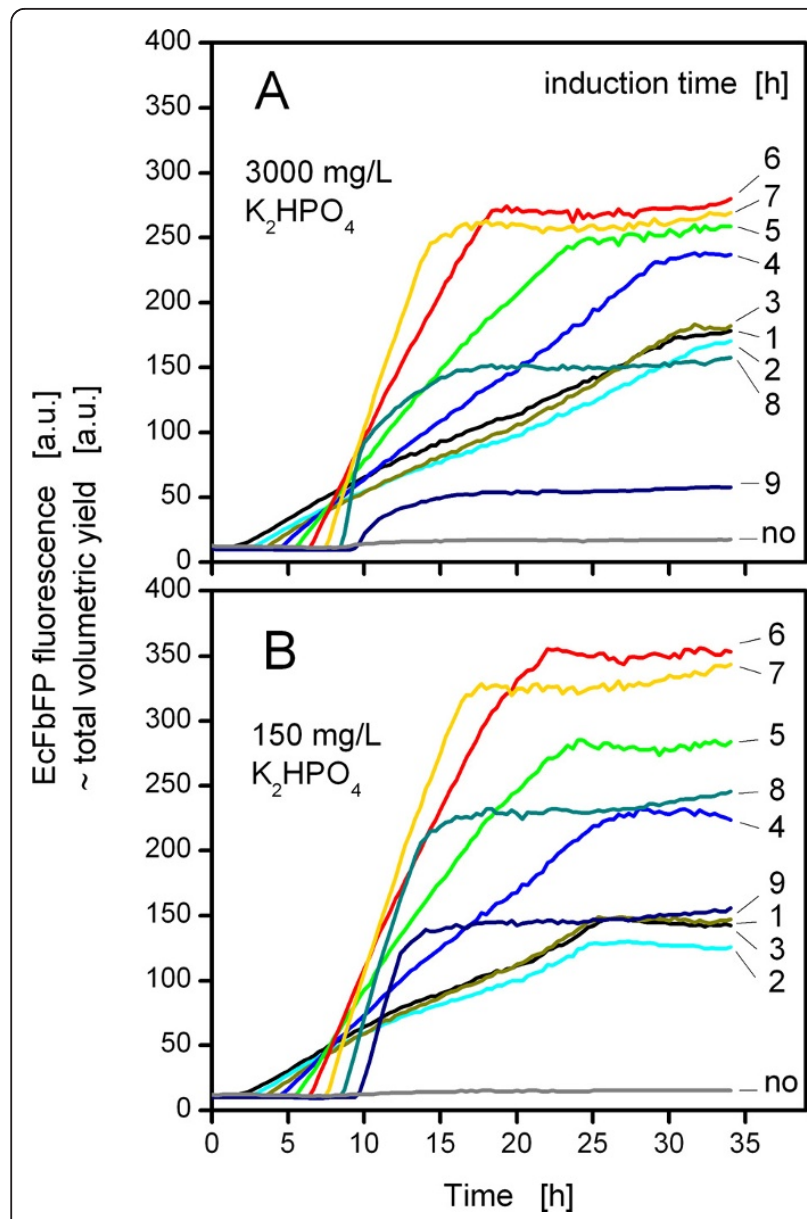

Figure 5 Influence of induction time on formation of EcFbFP under (A) unlimited $\left(3000 \mathrm{mg} / \mathrm{L} \mathrm{K}_{2} \mathrm{HPO}_{4}\right)$ and (B) limited (150 $\mathrm{mg} / \mathrm{L} \mathrm{K}_{2} \mathrm{HPO}_{4}$ ) conditions. The measurements were performed with the Robo-Lector with a signal gain factor of 70. Each curve represents the EcFbFP production from duplicate cultures $(n=2)$ for every different time of induction. The relative standard deviation of each curve did not exceed $\pm 10 \%$. The cultivation conditions were as follows: $37^{\circ} \mathrm{C}$, shaking diameter $3 \mathrm{~mm}$, shaking frequency $950 \mathrm{rpm}$, filling volume $200 \mu \mathrm{L}$, modified Wilms \& Reuss medium (7.5 g/L Glucose), induction with IPTG (final concentration $0.1 \mathrm{mM}$ ) at 1 to $9 \mathrm{~h}$.

visible for the different cultures. Noteworthy, the slope of this increase was steeper, the later the cells had been induced. This could be explained by the increased biomass at the later times of induction. The maximum EcFbFP production was achieved with an induction after $6 \mathrm{~h}$. Afterwards, the protein expression decreased dramatically, probably because the medium became exhausted for glucose as already discussed for Figure 4 . Similar effects resulted from inducing phosphate-limited cultures (Figure 5B), although here, in most cases, a higher EcFbFP fluorescence was observed than in unlimited cultures. To compare phosphate-unlimited and -limited conditions, the values (at $34 \mathrm{~h}$ ) for the EcFbFP fluorescence at the different induction points were plotted in Figure 6A. The phosphate-limited and unlimited cultures displayed a nearly constant EcFbFP production until $3 \mathrm{~h}$ of induction time. After that, both phosphate conditions showed a peak in EcFbFP fluorescence at $6 \mathrm{~h}$, whereas the phosphate-limited cultures reached higher values than the unlimited cultures. When cells were induced earlier than $4 \mathrm{~h}$ after inoculation, then the phosphate-limited cultures showed a lower EcFbFP expression. This could be explained by comparing the scattered light intensity with the specific productivity. Up to $4 \mathrm{~h}$ the biomass yield of both conditions was comparable (Figure 6B), though the specific productivity of the phosphate-limited culture was lower than for phosphate-unlimited conditions (Figure 6C), leading to a decreased overall expression (Figure 6A). After the $4 \mathrm{~h}$ induction point the specific productivity of the phosphate-limited cultures was significantly higher (Figure 6C) and together with only slightly less biomass (Figure 6B) resulted in more EcFbFP yield (Figure 6A).

In summary, the optimal time of induction could efficiently be determined with the applied experimental setup

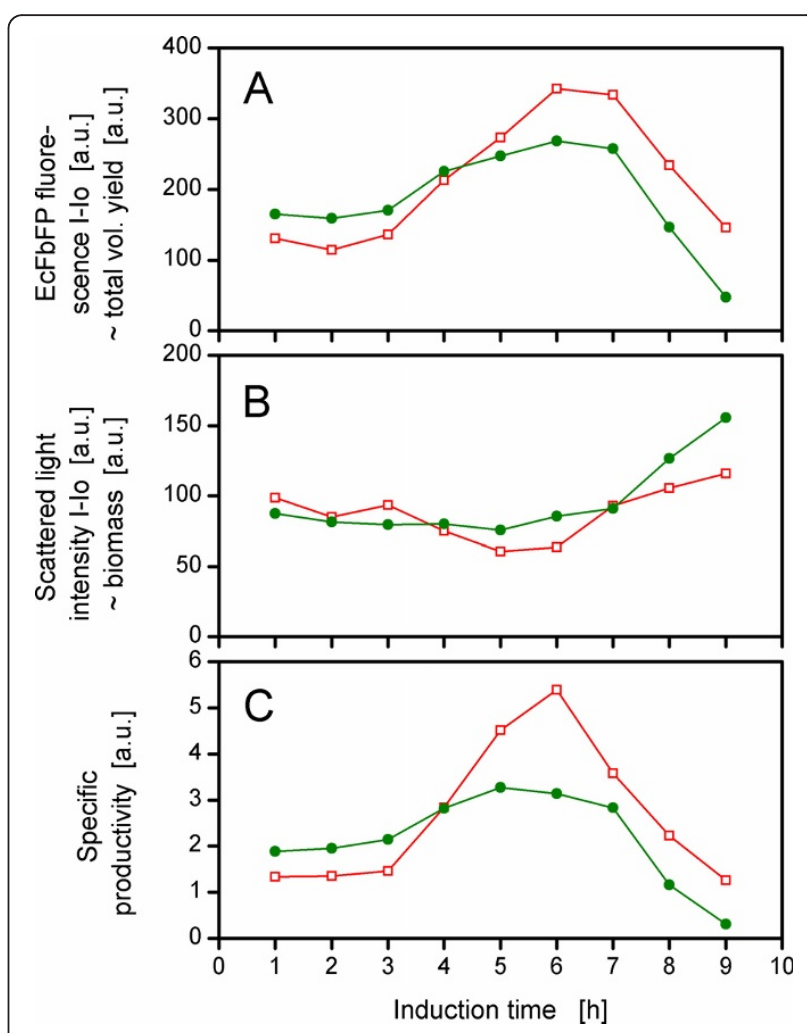

Figure 6 Influence of induction time for phosphate-unlimited (3000 mg/L K $\mathrm{HPO}_{4},{ }^{\circ}$ ) and phosphate-limited conditions (150 $\mathrm{mg} / \mathrm{L} \mathrm{K}_{2} \mathrm{HPO}_{4}, \square$ ) on (A) EcFbFP expression, (B) biomass yield and (C) specific productivity. For experimental setup (growth conditions etc.), refer to Figure 5; data taken from experiment of Figure 5 at 34 h of cultivation. 
and revealed that the induction time was the predominant parameter for the overall expression yield. Furthermore it became obvious that phosphate limitation could only further enhance product formation when an appropiate induction time was chosen. These effects could easily be investigated with the Robo-Lector platform.

\section{Verification of results in shake flasks}

To verify the results from the previous experiments, a cultivation in shake flasks was conducted under different phosphate-limited (225 mg/L, $150 \mathrm{mg} / \mathrm{L}, 75 \mathrm{mg} / \mathrm{L})$ and -unlimited conditions $(3000 \mathrm{mg} / \mathrm{L})$. The induction conditions were the same as in the Robo-Lector test with induction after $6 \mathrm{~h}$, as this was the optimal time identified previously (Figure 6). After $26 \mathrm{~h}$, samples from the different flasks were taken and the OD, scattered light and EcFbFP fluorescence was measured offline. Furthermore, samples were taken for SDS-PAGE and subsequent densitometric analysis. The results of these experiments were summarized in Figure 7.

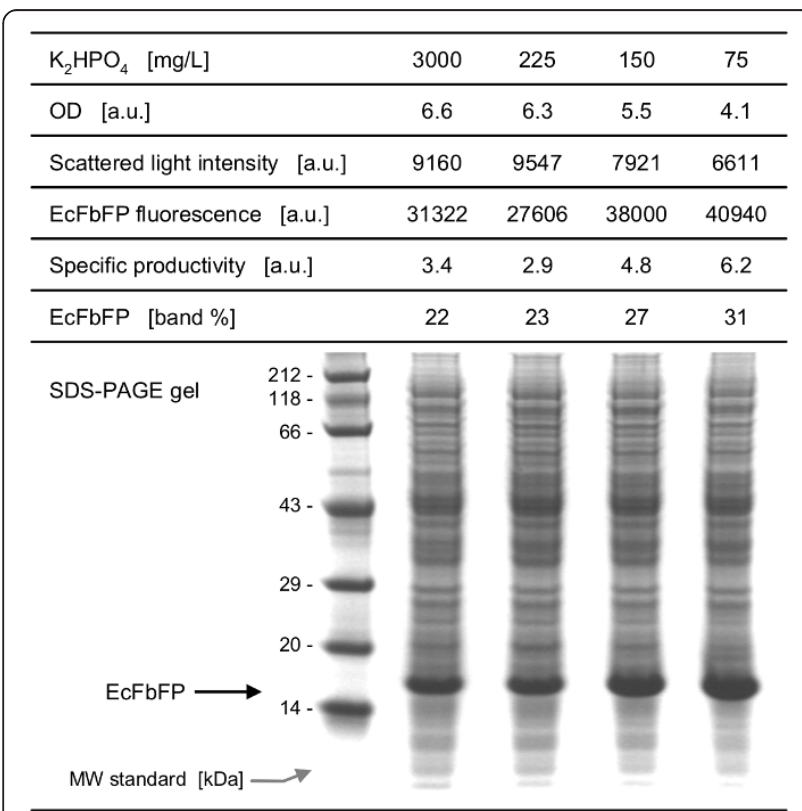

Figure 7 Comparison of phosphate-limited and -unlimited conditions in shake flasks. Comparison concerning biomass (OD and scattered light), product formation (ECFbFP fluorescence and SDS-PAGE gel) and specific productivity (fluorescence per scattered light and percent of EcFbFP band). Data from shake flask cultivations under the following conditions: $37^{\circ} \mathrm{C}$, shaking diameter $50 \mathrm{~mm}$, shaking frequency $350 \mathrm{rpm}$, filling volume $10 \mathrm{~mL}$, modified Wilms \& Reuss medium (7.5 g/L Glucose) with four different phosphate concentrations, induction with IPTG after $6 \mathrm{~h}$ (final concentration $0.1 \mathrm{mM}$ ), harvest time $26 \mathrm{~h}$. Samples for SDS-PAGE were normalized to a comparable protein content and EcFbFP bands were quantified with densitometry; MW means molecular weight standard. Measurement of scattered light intensity (signal gain factor 20) and EcFbFP fluorescence (signal gain factor 45) were conducted with the BioLector prototype.
The OD and scattered light intensity decreased with decreasing phosphate content of the medium (as could be expected from results of Figure 2). On the other hand, the EcFbFP fluorescence increased with decreasing phosphate, confirming the concept of increased product formation via a phosphate limitation in batch cultures (Figure 6). This was also indicated by the calculated specific productivity (EcFbFP fluorescence per scattered light), as this rose from 3.4 to 6.2 a.u. under phosphatelimited conditions. The percentage of the EcFbFP band per total protein content of the E.coli cells (band\%), as determined with SDS-PAGE and densitometry, confirmed this finding (increase from 22 to $31 \%$; see also SDS-PAGE gel).

\section{Conclusions}

In this study, different online-monitoring tools, such as RAMOS and BioLector, were used to study, characterize and optimize the given T7 expression system E.coli BL21(DE3) pRhotHi-2-EcFbFP. With these tools, the demand of the examined strain for phosphate as well as the time of the limitation onset could easily be determined. Furthermore, the effect of phosphate limitation on target protein production and the optimal time for induction was efficiently investigated by the automated high-throughput cultivation platform Robo-Lector. Lastly, the results were sucessfully verified by shake flask experiments.

These results revealed, that the phosphate limitation was suitable to redirect the available resources during cultivation (after 'enough' biomass has formed) to protein expression rather than in biomass production. This effect finally led to an increase in specific productivity of the target protein EcFbFP from $21 \%$ to $31 \%$ of total protein. To our knowledge, such an effect was for the first time shown for an IPTG-inducible expression system. This is attributed to the fact that cells can be metabolically active for a long time even under a phosphate limitation and the resulting stationary phase [43]. This principle was verified with RAMOS cultivations shown in Figure 3. Han and Lee also showed by proteome analysis that a subset of proteins involved in protein synthesis in $E$. coli was greatly enlarged during a phosphate limitation [10]. This, in turn, could increase protein synthesis capacity. Additionaly, a higher plasmid copy number was observed in a non-induced fed-batch culture because of a phosphate limitation [44]. This might lead to higher amounts of mRNA for the target protein and consequently to a higher product yield in induced cultures. Furthermore, the aspects described here are most probably also the main reason why host/vector systems using the phosphate starvation-inducible promoter phoA show high productivities, as they tend to be very efficient expression systems $[9,17]$. 
Increasing the specific productivity by phosphate limitation can be especially important in high-throughput cultivations such as MTP-based processes. Since in MTPs the cultivation volume is mostly fixed and the biomass often can not be increased due to oxygen limitations, the only way to increase the total productivity is to raise the specific productivity [1]. A promising idea would also be to combine the presented online-monitoring tools with 'omics'-techniques to further investigate the described effects of phosphate limitation, as the exact molecular reasons for the increased specific productivity remain unclear. This could help to find new targets to further enhance the production capacity of recombinant E.coli-strains.

\section{Acknowledgements}

We are grateful for the financial support of the Stiftung Industrieforschung (Köln, Germany) for the project S735. Further we want to thank K. Kottmeier, T. Drepper, M. Funke, M. Scheidle and T. Palmen for helpful discussions, M.J. Blümich and N. Chen for proofreading the manuscript and R. Petri for sample analyses.

\section{Authors' contributions}

$\mathrm{RH}$ designed and coordinated the study and prepared the manuscript. SR and NR performed cultivation experiments. JB assisted with data analysis and manuscript preparation. All authors read and approved the final manuscript.

\section{Competing interests}

The authors declare that they have no competing interests.

Received: 10 May 2010 Accepted: 17 March 2011

Published: 17 March 2011

\section{References}

1. Markland K: Methodology for high-throughput production of soluble recombinant proteins in Escherichia coli. Royal Institute of Technology (KTH), Stockholm, Sweden 2006, ISBN 978-91-7178-561-9.

2. Donovan RS, Robinson CW, Glick BR: Review: optimizing inducer and culture conditions for expression of foreign proteins under the control of the lac promoter. J Ind Microbiol 1996, 16(3):145-154.

3. Flickinger MC, Rouse MP: Sustaining protein synthesis in the absence of rapid cell division: an investigation of plasmid-encoded protein expression in Escherichia coli during very slow growth. Biotechnol Prog 1993, 9(6):555-572.

4. Matin A: The molecular basis of carbon-starvation-induced general resistance in Escherichia coli. Mol Microbiol 1991, 5(1):3-10.

5. Tunner JR, Robertson CR, Schippa S, Matin A: Use of glucose starvation to limit growth and induce protein production in Escherichia coli. Biotechnol Bioeng 1992, 40(2):271-279.

6. Rowe DC, Summers DK: The quiescent-cell expression system for protein synthesis in Escherichia coli. Appl Environ Microbiol 1999, 65(6):2710-2715.

7. Mukherjee KJ, Rowe DC, Watkins NA, Summers DK: Studies of single-chain antibody expression in quiescent Escherichia coli. Appl Environ Microbiol 2004, 70(5):3005-3012.

8. Summers DK, Chant E: Chemical induction in quiescence in bacteria. Patent US2009/0004700A1 2009.

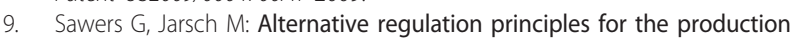
of recombinant proteins in Escherichia coli. Appl Microbiol Biotechnol 1996, 46(1):1-9.

10. Han MJ, Lee SY: The Escherichia coli proteome: past, present, and future prospects. Microbiol Mol Biol Rev 2006, 70(2):362-439.

11. Peterson CN, Mandel MJ, Silhavy TJ: Escherichia coli starvation diets: essential nutrients weigh in distinctly. J Bacteriol 2005, 187(22):7549-7553.
12. Vila P, Luis Corchero J, Cubarsi R, Villaverde A: Enhanced fitness of recombinant protein synthesis in the stationary phase of Escherichia coli batch cultures. Biotechnol Lett 1997, 19(3):225-228.

13. Bentley WE, Mirjalili N, Andersen DC, Davis RH, Kompala DS: Plasmidencoded protein: The principal factor in the "metabolic burden" associated with recombinant bacteria. Biotechnol Bioeng 1990, 35(7):668-681.

14. Glick BR: Metabolic load and heterologous gene expression. Biotechnol Adv 1995, 13(2):247-261.

15. Champion KM, Nishihara JC, Joly JC, Arnott D: Similarity of the Escherichia coli proteome upon completion of different biopharmaceutical fermentation processes. Proteomics 2001, 1(9):1133-1148.

16. Wanner BL: Phosphorus assimilation and control of the phosphate regulon. Escherichia coli and Salmonella: cellular and molecular biology. 2 edition. ASM Press, Washington, DC; 1996, 1357-1381.

17. Lübke $C$, Boidol W, Petri T: Analysis and optimization of recombinant protein production in Escherichia coli using the inducible pho A promoter of the E. coli alkaline phosphatase. Enzyme Microb Technol 1995, 17(10):923-928

18. Shin PK, Seo JH: Analysis of E. coli phoA-lacZ fusion gene expression inserted into a multicopy plasmid and host cell's chromosome. Biotechnol Bioeng 1990, 36(11):1097-1104.

19. Kottmeier K, Müller C, Huber R, Büchs J: Increased product formation caused by a directed secondary substrate limitation in a batch Hansenula polymorpha culture. Appl Microbiol Biotechnol 2009.

20. Ryan W, Parulekar SJ, Stark BC: Expression of B-lactamase by recombinant Escherichia coli strains containing plasmids of different sizes-effects of $\mathrm{pH}$, phosphate, and dissolved oxygen. Biotechnol Bioeng 1989, 34(3):309-319.

21. Van Dien SJ, Keasling JD: Control of polyphosphate metabolism in genetically engineered Escherichia coli. Enzyme Microb Technol 1999, 24(12):21-25.

22. Torriani A: From cell membrane to nucleotides: the phosphate regulon in Escherichia coli. Bioessays 1990, 12(8):371-376.

23. Schurdell MS, Woodbury GM, McCleary WR: Genetic evidence suggests that the intergenic region between pstA and pstB plays a role in the regulation of rpoS translation during phosphate limitation. $J$ Bacteriol 2007, 189(3):1150-1153.

24. VanBogelen RA, Olson ER, Wanner BL, Neidhardt FC: Global analysis of proteins synthesized during phosphorus restriction in Escherichia coli. J Bacteriol 1996, 178(15):4344-4366.

25. Jensen EB, Carlsen S: Production of recombinant human growth hormone in Escherichia coli: expression of different precursors and physiological effects of glucose, acetate, and salts. Biotechnol Bioeng 1990, 36(1):1-11.

26. Johansson L, Lindskog A, Silfversparre G, Cimander C, Nielsen KF, Liden G Shikimic acid production by a modified strain of E. coli (W3110. shik1) under phosphate-limited and carbon-limited conditions. Biotechnol Bioeng 2005, 92(5):541-552.

27. De Hollander JA, Eswilder FR, Noordover JAC: Amino acid fermentation processes. Patent US5763230 1998.

28. Anderlei T, Büchs J: Device for sterile online measurement of the oxygen transfer rate in shaking flasks. Biochem Eng J 2001, 7(2):157-162.

29. Anderlei T, Zang $W$, Papaspyrou $M$, Büchs J: Online respiration activity measurement (OTR, CTR, RQ) in shake flasks. Biochem Eng J 2004, 17(3):187-194.

30. Samorski M, Müller-Newen G, Büchs J: Quasi-continuous combined scattered light and fluorescence measurements: a novel measurement technique for shaken microtiter plates. Biotechnol Bioeng 2005, 92(1):61-68.

31. Kensy F, Zang E, Faulhammer C, Tan RK, Büchs J: Validation of a highthroughput fermentation system based on online monitoring of biomass and fluorescence in continuously shaken microtiter plates. Microb Cell Fact 2009, 8(1):31.

32. Huber R, Ritter D, Hering T, Hillmer AK, Kensy F, Müller C, Wang L, Büchs J: Robo-Lector - a novel platform for automated high-throughput cultivations in microtiter plates with high information content. Microb Cell Fact 2009, 8(42).

33. Katzke N, Arvani S, Bergmann R, Circolone F, Markert A, Svensson V, Jaeger KE, Heck A, Drepper T: A novel T7 RNA polymerase dependent 
expression system for high-level protein expression in the phototrophic bacterium Rhodobacter capsulatus. Protein Expr Purif 2009.

34. Scheidle M, Klinger J, Büchs J: Combination of on-line $\mathrm{pH}$ and oxygen transfer rate measurement in shake flasks by fiber optical technique and respiration activity monitoring system (RAMOS). Sensors 2007, 7:3472-3480

35. Wilms B, Hauck A, Reuss M, Syldatk C, Mattes R, Siemann M, Altenbuchner J: High-cell-density fermentation for production of L-Ncarbamoylase using an expression system based on the Escherichia coli rhaBAD promoter. Biotechnol Bioeng 2001, 73(2):95-103.

36. Drepper T, Huber R, Heck A, Circolone F, Hillmer AK, Büchs J, Jaeger KE: Flavin mononucleotide-based fluorescent reporter proteins outperform green fluorescent protein-like proteins as quantitative in vivo real-time reporters. Appl Environ Microbiol 2010, 76(17):5990-5994.

37. Drepper T, Eggert T, Circolone F, Heck A, Krauss U, Guterl JK, Wendorff M, Losi A, Gartner W, Jaeger KE: Reporter proteins for in vivo fluorescence without oxygen. Nat Biotechnol 2007, 25(4):443-445.

38. Seletzky JM, Noack U, Hahn S, Knoll A, Amoabediny G, Büchs J: An experimental comparison of respiration measuring techniques in fermenters and shake flasks: exhaust gas analyzer vs. RAMOS device vs. respirometer. J Ind Microbiol Biotechnol 2007, 34(2):123-130.

39. Damoglou AP, Dawes EA: Studies on the lipid content and phosphate requirement of glucose-and acetate-grown Escherichia coli. Portland Press Ltd; 1968:110:775.

40. Wang Y, Ding H, Du P, Gan R, Ye Q: Production of phoA promotercontrolled human epidermal growth factor in fed-batch cultures of Escherichia coli YK537 (pAET-8). Process Biochemistry 2005, 40(9):3068-3074.

41. Jeude M, Dittrich B, Niederschulte H, Anderlei T, Knocke C, Klee D, Büchs J: Fed-batch mode in shake flasks by slow-release technique. Biotechnol Bioeng 2006, 95(3):433-445.

42. Losen M, Frölich B, Pohl M, Büchs J: Effect of oxygen limitation and medium composition on Escherichia coli fermentation in shake-flask cultures. Biotechnol Prog 2004, 20(4):1062-1068.

43. Moreau PL, Gerard F, Lutz NW, Cozzone P: Non-growing Escherichia coli cells starved for glucose or phosphate use different mechanisms to survive oxidative stress. Molecular Microbiology 2001, 39(4):1048-1060.

44. Horn U, Krug M, Sawistowski J: Effect of high density cultivation on plasmid copy number in recombinant Escherichia coli cells. Biotechnol Lett 1990, 12(3):191-196.

doi:10.1186/1472-6750-11-22

Cite this article as: Huber et al:: Utilizing high-throughput experimentation to enhance specific productivity of an E.coli T7 expression system by phosphate limitation. BMC Biotechnology 201 11:22.

\section{Submit your next manuscript to BioMed Central and take full advantage of:}

- Convenient online submission

- Thorough peer review

- No space constraints or color figure charges

- Immediate publication on acceptance

- Inclusion in PubMed, CAS, Scopus and Google Scholar

- Research which is freely available for redistribution 